\title{
SUMSETS CONTAINED IN SETS OF UPPER BANACH DENSITY 1
}

\author{
MELVYN B. NATHANSON
}

\begin{abstract}
Every set $A$ of positive integers with upper Banach density 1 contains an infinite sequence of pairwise disjoint subsets $\left(B_{i}\right)_{i=1}^{\infty}$ such that $B_{i}$ has upper Banach density 1 for all $i \in \mathbf{N}$ and $\sum_{i \in I} B_{i} \subseteq A$ for every nonempty finite set $I$ of positive integers.
\end{abstract}

\section{UPPER BANACH DENSITY}

Let $\mathbf{N}, \mathbf{N}_{0}$, and $\mathbf{Z}$ denote, respectively, the sets of positive integers, nonnegative integers, and integers. Let $|S|$ denote the cardinality of the set $S$. We define the interval of integers

$$
[x, y]=\{n \in \mathbf{N}: x \leq n \leq y\} .
$$

Let $A$ be a set of positive integers. Let $n \in \mathbf{N}$. For all $u \in \mathbf{N}_{0}$, we have

$$
|A \cap[u, u+n-1]| \in[0, n]
$$

and so

$$
f_{A}(n)=\max _{u \in \mathbf{N}_{0}}|A \cap[u, u+n-1]|
$$

exists. The upper Banach density of $A$ is

$$
\delta(A)=\limsup _{n \rightarrow \infty} \frac{f_{A}(n)}{n} .
$$

Let $n_{1}, n_{2} \in \mathbf{N}$. There exists $u_{1}^{*} \in \mathbf{N}_{0}$ such that, with $u_{2}^{*}=u_{1}^{*}+n_{1}$,

$$
\begin{aligned}
f_{A}\left(n_{1}+n_{2}\right) & =\left|A \cap\left[u_{1}^{*}, u_{1}^{*}+n_{1}+n_{2}-1\right]\right| \\
& =\left|A \cap\left[u_{1}^{*}, u_{1}^{*}+n_{1}-1\right]\right|+\left|A \cap\left[u_{1}^{*}+n_{1}, u_{1}^{*}+n_{1}+n_{2}-1\right]\right| \\
& =\left|A \cap\left[u_{1}^{*}, u_{1}^{*}+n_{1}-1\right]\right|+\left|A \cap\left[u_{2}^{*}, u_{2}^{*}+n_{2}-1\right]\right| \\
& \leq f_{A}\left(n_{1}\right)+f_{A}\left(n_{2}\right) .
\end{aligned}
$$

It is well known, and proved in the Appendix, that this inequality implies that

$$
\delta(A)=\lim _{n \rightarrow \infty} \frac{f_{A}(n)}{n}=\inf _{n \in \mathbf{N}} \frac{f_{A}(n)}{n} .
$$

Date: October 6, 2018.

2010 Mathematics Subject Classification. 11B05, 11B13, $11 \mathrm{~B} 75$.

Key words and phrases. Sumsets, Banach density, additive number theory, Ramsay theory.

Supported in part by a grant from the PSC-CUNY Research Award Program. 


\section{An ERdős SUMSET CONJECTURE}

About 40 years ago, Erdős conjectured that if $A$ is a set of positive integers of positive upper Banach density, then there exist infinite sets $B$ and $C$ of positive integers such that $B+C \subseteq A$. This conjecture has not yet been verified or disproved.

The translation of the set $X$ by $t$ is the set

$$
X+t=\{x+t: x \in X\} .
$$

Let $B$ and $C$ be sets of integers. For every integer $t$, if $B^{\prime}=B+t$ and $C^{\prime}=C-t$, then

$$
B^{\prime}+C^{\prime}=(B+t)+(C-t)=B+C .
$$

In particular, if $C$ is bounded below and $t=\min (C)$, then $0=\min \left(C^{\prime}\right)$ and $B^{\prime} \subseteq B^{\prime}+C^{\prime}$. It follows that if $B$ and $C$ are infinite sets such that $B+C \subseteq A$, then, by translation, there exist infinite sets $B^{\prime}$ and $C^{\prime}$ such that $B^{\prime} \subseteq A$ and $B^{\prime}+C^{\prime} \subseteq A$.

However, a set $A$ with positive upper Banach density does not necessarily contain infinite subsets $B$ and $C$ with $B+C \subseteq A$. For example, let $A$ be any set of odd numbers. For all sets $B$ and $C$ of odd numbers, the sumset $B+C$ is a set of even numbers, and so $A \cap(B+C)=\emptyset$. Of course, in this example we have $B+C \subseteq A+1$.

In this note we prove that if $A$ is a set of positive integers with upper Banach density $\delta(A)=1$, then for every $h \geq 2$ there exist pairwise disjoint subsets $B_{1}, \ldots, B_{h}$ of $A$ such that $\delta\left(B_{i}\right)=1$ for all $i=1, \ldots, h$ and

$$
B_{1}+\cdots+B_{h} \subseteq A .
$$

Indeed, Theorem 2 states an even stronger result.

There are sets $A$ of upper Banach density 1 for which no infinite subset $B$ of $A$ satisfies $2 B \subseteq A+t$ for any integer $t$. A simple example is

$$
A=\bigcup_{i=1}^{\infty}\left[4^{i}, 4^{i}+i-1\right] .
$$

The set $A$ is the union of the infinite sequence of pairwise disjoint intervals

$$
A_{i}=\left[4^{i}, 4^{i}+i-1\right] .
$$

Let $t \in \mathbf{N}_{0}$. There exists $i_{0}(t)$ such that $4^{i}-i>t$ for all $i \geq i_{0}(t)$. If $b_{i} \in A_{i}$ for some $i \geq i_{0}(t)$, then

$$
4^{i}+i+t<2 \cdot 4^{i} \leq 2 b_{i}<2 \cdot 4^{i}+2 i<4^{i+1}-2 t \leq 4^{i+1}-t
$$

and so $2 b_{i} \notin 2 A \pm t$. If $B$ is an infinite subset of $A$, then for infinitely many $i$ there exist integers $b_{i} \in B \cap A_{i}$, and so $2 B \nsubseteq A+t$ for all $t \in \mathbf{Z}$.

There are very few results about the Erdös conjecture. In 1980, Nathanson [9] proved that if $\delta(A)>0$, then for every $n$ there is a finite set $C$ with $|C|=n$ and a subset $B$ of $A$ with $\delta(B)>0$ such that $B+C \subseteq A$. In 2015, Di Nasso, Goldbring, Jin, Leth, Lupini, and Mahlburg [3] used nonstandard analysis to prove that the Erdős conjecture is true for sets $A$ with upper Banach density $\delta(A)>1 / 2$. They also proved that if $\delta(A)>0$, then there exist infinite sets $B$ and $C$ and an integer $t$ such that

$$
B+C \subseteq A \cup(A+t) .
$$

It would be of interest to have purely combinatorial proofs of the results of $\mathrm{Di}$ Nasso, et al. 
For related work, see Di Nasso [1, 2], Gromov [4, Hegyvári [5] 6, Hindman [7, and Jin 8 .

\section{Results}

The following result is well known.

Lemma 1. A set of positive integers has upper Banach density 1 if and only if, for every $d$, it contains infinitely many pairwise disjoint intervals of $d$ consecutive integers.

Proof. Let $A$ be a set of positive integers. If, for every positive integer $d$, the set $A$ contains an interval of $d$ consecutive integers, then

$$
\max _{u \in \mathbf{N}_{0}}\left(\frac{|A \cap[u, u+d-1]|}{d}\right)=1
$$

and so

$$
\delta(A)=\lim _{d \rightarrow \infty} \max _{u \in \mathbf{N}_{0}}\left(\frac{|A \cap[u, u+d-1]|}{d}\right)=1 .
$$

Suppose that, for some integer $d \geq 2$, the set $A$ contains no interval of $d$ consecutive integers. For every $u \in \mathbf{N}_{0}$, we consider the interval $I_{u, n}=[u, u+n-1]$. By the division algorithm, there are integers $q$ and $r$ with $0 \leq r<d$ such that

$$
\left|I_{u, n}\right|=n=q d+r
$$

and

$$
q=\frac{n-r}{d}>\frac{n}{d}-1
$$

For $j=1, \ldots, q$, the intervals of integers

$$
I_{u, n}^{(j)}=[u+(j-1) d, u+j d-1]
$$

and

$$
I_{u, n}^{(q+1)}=[u+q d, u+n-1]
$$

are pairwise disjoint subsets of $I_{u, n}$ such that

$$
I_{u, n}=\bigcup_{j=1}^{q+1} I_{u, n}^{(j)} .
$$

We have

$$
A \cap I_{u, n}=\bigcup_{j=1}^{q+1}\left(A \cap I_{u, n}^{(j)}\right)
$$

If $A$ contains no interval of $d$ consecutive integers, then, for all $j \in[1, q]$, at least one element of the interval $I_{u, n}^{(j)}$ is not an element of $A$, and so

$$
\left|A \cap I_{u, n}^{(j)}\right| \leq\left|I_{u, n}^{(j)}\right|-1 .
$$


It follows that

$$
\begin{aligned}
\left|A \cap I_{u, n}\right| & =\sum_{j=1}^{q+1}\left|A \cap I_{u, n}^{(j)}\right| \leq \sum_{j=1}^{q}\left(\left|I_{u, n}^{(j)}\right|-1\right)+\left|I_{u, n}^{(q+1)}\right| \\
& =\sum_{j=1}^{q+1}\left|I_{u, n}^{(j)}\right|-q=\left|I_{u, n}\right|-q=n-q \\
& <n-\frac{n}{d}+1=\left(1-\frac{1}{d}\right) n+1 .
\end{aligned}
$$

Dividing by $n=\left|I_{u, n}\right|$, we obtain

$$
\max _{u \in \mathbf{N}_{0}} \frac{\left|A \cap I_{u, n}\right|}{n} \leq 1-\frac{1}{d}+\frac{1}{n} .
$$

and so

$$
\delta(A)=\lim _{n \rightarrow \infty} \max _{u \in \mathbf{N}_{0}} \frac{\left|A \cap I_{u, n}\right|}{n} \leq 1-\frac{1}{d}<1
$$

which is absurd. Therefore, $A$ contains an interval of $d$ consecutive integers for every $d \in \mathbf{N}$.

To prove that $A$ contains infinitely many intervals of size $d$, it suffices to prove that if $[u, u+d-1] \subseteq A$, then $[v, v+d-1] \subseteq A$ for some $v \geq u+d$. Let $d^{\prime}=u+2 d$. There exists $u^{\prime} \in \mathbf{N}$ such that

$$
\left[u^{\prime}, u^{\prime}+d^{\prime}-1\right]=\left[u^{\prime}, u^{\prime}+u+2 d-1\right] \subseteq A .
$$

Choosing $v=u^{\prime}+u+d$, we have $v \geq u+d$ and

$$
[v, v+d-1] \subseteq\left[u^{\prime}, u^{\prime}+u+2 d-1\right] \subseteq A .
$$

This completes the proof.

Let $\mathcal{F}(S)$ denote the set of all finite subsets of the set $S$, and let $\mathcal{F}^{*}(S)$ denote the set of all nonempty finite subsets of $S$. We have the fundamental binomial identity

$$
\mathcal{F}^{*}([1, n+1])=\mathcal{F}^{*}([1, n]) \cup\{\{n+1\} \cup J: J \in \mathcal{F}([1, n])\} .
$$

Theorem 1. Let $A$ be a set of positive integers that has upper Banach density 1. For every sequence $\left(\ell_{j}\right)_{j=1}^{\infty}$ of positive integers, there exists a sequence $\left(b_{j}\right)_{j=1}^{\infty}$ of positive integers such that

$$
b_{j+1} \geq b_{j}+\ell_{j}
$$

for all $j \in \mathbf{N}$, and

$$
\sum_{j \in J}\left[b_{j}, b_{j}+\ell_{j}-1\right] \subseteq A
$$

for all $J \in \mathcal{F}^{*}(\mathbf{N})$.

Proof. We shall construct the sequence $\left(b_{j}\right)_{j=1}^{\infty}$ by induction. For $n=1$, choose $b_{1} \in A$ such that $\left[b_{1}, b_{1}+\ell_{1}-1\right] \subseteq A$.

Suppose that $\left(b_{j}\right)_{j=1}^{n}$ is a finite sequence of positive integers such that $b_{j+1} \geq$ $b_{j}+\ell_{j}$ for $j \in[1, n-1]$ and

$$
\sum_{j \in J}\left[b_{j}, b_{j}+\ell_{j}-1\right] \subseteq A
$$


for all $J \in \mathcal{F}^{*}([1, n])$. By Lemma 1, there exists $b_{n+1} \in A$ such that

$$
b_{n+1} \geq b_{n}+\ell_{n}
$$

and

$$
\left[b_{n+1}, \sum_{j=1}^{n+1}\left(b_{j}+\ell_{j}\right)-1\right] \subseteq A
$$

It follows that

$$
\left[b_{n+1}, b_{n+1}+\ell_{n+1}-1\right] \subseteq A .
$$

Let $J \in \mathcal{F}([1, n])$. If

$$
\begin{aligned}
a & \in \sum_{j \in\{n+1\} \cup J}\left[b_{j}, b_{j}+\ell_{j}-1\right] \\
& =\left[b_{n+1}, b_{n+1}+\ell_{n+1}-1\right]+\sum_{j \in J}\left[b_{j}, b_{j}+\ell_{j}-1\right]
\end{aligned}
$$

then

$$
\begin{aligned}
b_{n+1} \leq a & \leq\left(b_{n+1}+\ell_{n+1}-1\right)+\sum_{j \in J}\left(b_{j}+\ell_{j}-1\right) \\
& \leq \sum_{j=1}^{n+1}\left(b_{j}+\ell_{j}\right)-1
\end{aligned}
$$

and so $a \in A$ and

$$
\sum_{j \in\{n+1\} \cup J}\left[b_{j}, b_{j}+\ell_{j}-1\right] \subseteq\left[b_{n+1}, \sum_{j=1}^{n+1}\left(b_{j}+\ell_{j}\right)-1\right] \subseteq A .
$$

Relations (11), (2), and (3) imply that

$$
\sum_{j \in J}\left[b_{j}, b_{j}+\ell_{j}-1\right] \subseteq A
$$

for all $J \in \mathcal{F}^{*}([1, n+1])$. This completes the induction.

Theorem 2. Every set $A$ of positive integers that has upper Banach density 1 contains an infinite sequence of pairwise disjoint subsets $\left(B_{i}\right)_{i=1}^{\infty}$ such that $B_{i}$ has upper Banach density 1 for all $i \in \mathbf{N}$ and

$$
\sum_{i \in I} B_{i} \subseteq A
$$

for all $I \in \mathcal{F}^{*}(\mathbf{N})$.

Proof. Let $\left(\ell_{j}\right)_{j=1}^{\infty}$ be a sequence of positive integers such that $\lim _{j \rightarrow \infty} \ell_{j}=\infty$, and let $\left(b_{j}\right)_{j=1}^{\infty}$ be a sequence of positive integers that satisfies Theorem 11. (For simplicity, we can let $\ell_{j}=j$ for all $j$.) Let $\left(X_{i}\right)_{i=1}^{\infty}$ be a sequence of infinite sets of positive integers that are pairwise disjoint. For $i \in \mathbf{N}$, let

$$
B_{i}=\bigcup_{j \in X_{i}}\left[b_{j}, b_{j}+\ell_{j}-1\right] .
$$

The set $B_{i}$ contains intervals of $\ell_{j}$ consecutive integers for infinitely many $\ell_{j}$, and so $B_{i}$ has upper Banach density 1. 
Let $I \in \mathcal{F}^{*}(\mathbf{N})$. If

$$
a \in \sum_{i \in I} B_{i} \subseteq A
$$

then for each $i \in I$ there exists $a_{i} \in B_{i}$ such that $a=\sum_{i \in I} a_{i}$. If $a_{i} \in B_{i}$, then there exists $j_{i} \in X_{i}$ such that

$$
x_{i} \in\left[b_{j_{i}}, b_{j_{i}}+\ell_{j_{i}}-1\right] .
$$

We have $J=\left\{j_{i}: i \in I\right\} \in \mathcal{F}^{*}(\mathbf{N})$ and

$$
a \in \sum_{j_{i} \in J}\left[b_{j_{i}}, b_{j_{i}}+\ell_{j_{i}}-1\right] \subseteq A .
$$

This completes the proof.

Theorem 3. Let $A$ be a set of integers that contains arbitrarily long finite arithmetic progressions with bounded differences. There exist positive integers $m$ and $r$, and an infinite sequence of pairwise disjoint sets $\left(B_{i}\right)_{i=1}^{\infty}$ such that $B_{i}$ has upper Banach density 1 for all $i \in \mathbf{N}$ and

$$
m * \sum_{i \in I} B_{i}+r \subseteq A
$$

for all $I \in \mathcal{F}^{*}(\mathbf{N})$.

Proof. If the differences in the infinite set of finite arithmetic progressions contained in $A$ are bounded by $m_{0}$, then there exists a difference $m \leq m_{0}$ that occurs infinitely often. It follows that there are arbitrarily long finite arithmetic progressions with difference $m$. Because there are only finitely many congruence classes modulo $m$, there exists a congruence class $r(\bmod m)$ such that $A$ contains arbitrarily long sequences of consecutive integers in the the congruence class $r(\bmod m)$. Thus, there exists an infinite set $A^{\prime}$ such that

$$
m * A^{\prime}+r \subseteq A
$$

and $A^{\prime}$ contains arbitrarily long sequences of consecutive integers. Equivalently, $A^{\prime}$ has Banach density 1 . By Theorem 2, the sequence $A^{\prime}$ contains an infinite sequence of pairwise disjoint subsets $\left(B_{i}\right)_{i=1}^{\infty}$ such that $B_{i}$ has upper Banach density 1 for all $i \in \mathbf{N}$ and

$$
\sum_{i \in I} B_{i} \subseteq A^{\prime}
$$

for all $I \in \mathcal{F}^{*}(\mathbf{N})$. It follows that

$$
m * \sum_{i \in I} B_{i}+r \subseteq m * A^{\prime}+r \subseteq A
$$

for all $I \in \mathcal{F}^{*}(\mathbf{N})$. This completes the proof.

\section{Appendix A. Subadditivity And Limits}

A real-valued arithmetic function $f$ is subadditive if

$$
f\left(n_{1}+n_{2}\right) \leq f\left(n_{1}\right)+f\left(n_{2}\right)
$$

for all $n_{1}, n_{2} \in \mathbf{N}$.

The following result is sometimes called Fekete's lemma. 
Lemma 2. If $f$ is a subadditive arithmetic function, then $\lim _{n \rightarrow \infty} f(n) / n$ exists, and

$$
\lim _{n \rightarrow \infty} \frac{f(n)}{n}=\inf _{n \in \mathbf{N}} \frac{f(n)}{n} .
$$

Proof. It follows by induction from inequality (4) that

$$
f\left(n_{1}+\cdots+n_{q}\right) \leq f\left(n_{1}\right)+\cdots+f\left(n_{q}\right)
$$

for all $n_{1}, \ldots, n_{q} \in \mathbf{N}$. Let $f(0)=0$. Fix a positive integer $d$. For all $q, r \in \mathbf{N}_{0}$, we have

$$
f(q d+r) \leq q f(d)+f(r) .
$$

By the division algorithm, every nonnegative integer $n$ can be represented uniquely in the form $n=q d+r$, where $q \in \mathbf{N}_{0}$ and $r \in[0, d-1]$. Therefore,

$$
\frac{f(n)}{n}=\frac{f(q d+r)}{n} \leq \frac{q f(d)}{q d}+\frac{f(r)}{n}=\frac{f(d)}{d}+\frac{f(r)}{n} .
$$

Because the set $\{f(r): r \in[0, d-1]\}$ is bounded, it follows that

$$
\limsup _{n \rightarrow \infty} \frac{f(n)}{n} \leq \limsup _{n \rightarrow \infty}\left(\frac{f(d)}{d}+\frac{f(r)}{n}\right)=\frac{f(d)}{d}
$$

for all $d \in \mathbf{N}$, and so

$$
\limsup _{n \rightarrow \infty} \frac{f(n)}{n} \leq \inf _{d \in \mathbf{N}} \frac{f(d)}{d} \leq \liminf _{d \rightarrow \infty} \frac{f(d)}{d}=\liminf _{n \rightarrow \infty} \frac{f(n)}{n} .
$$

This completes the proof.

\section{REFERENCES}

[1] M. Di Nasso, An elementary proof of Jin's theorem with a bound, Electron. J. Combin. 21 (2014), no. 2, Paper 2.37, 7.

[2] — Embeddability properties of difference sets, Integers 14 (2014), Paper No. A27, 24.

[3] M. Di Nasso, I. Goldbring, R. Jin, S. Leth, M. Lupini, and K. Mahlburg, On a sumset conjecture of Erdös, Canad. J. Math. 67 (2015), no. 4, 795-809.

[4] M. L. Gromov, Colorful categories, Uspekhi Mat. Nauk 70 (2015), no. 4(424), 3-76.

[5] N. Hegyvári, On the dimension of the Hilbert cubes, J. Number Theory 77 (1999), no. 2, $326-330$.

[6] - On additive and multiplicative Hilbert cubes, J. Combin. Theory Ser. A 115 (2008), no. $2,354-360$.

[7] N. Hindman, Ultrafilters and combinatorial number theory, Number theory, Carbondale 1979 (Proc. Southern Illinois Conf., Southern Illinois Univ., Carbondale, Ill., 1979), Lecture Notes in Math., vol. 751, Springer, Berlin, 1979, pp. 119-184.

[8] R. Jin, Standardizing nonstandard methods for upper Banach density problems, Unusual Applications of Number Theory, DIMACS Ser. Discrete Math. Theoret. Comput. Sci., vol. 64, Amer. Math. Soc., Providence, RI, 2004, pp. 109-124.

[9] M. B. Nathanson, Sumsets contained in infinite sets of integers, J. Combin. Theory Ser. A 28 (1980), no. 2, 150-155.

Lehman College (CUNY),Bronx, New York 10468

E-mail address: melvyn.nathanson@lehman.cuny.edu 